\title{
DEL AUTOR COMO ESPECTRO O DE LA FANTOMAQUIA. DEL ARTE DE LIDIAR (CON) FANTASMAS
}

\author{
Begonya SÁEZ TAJAFUERCE \\ Universitat Autònoma de Barcelona
}

Escribo para borrar mi nombre Georges Bataille

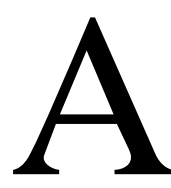
mediados del siglo Xx, Roland Barthes de un lado y Michel Foucault, de otro, en un conciso y breve $\mathrm{y}$, sin embargo, determinante ejercicio de interlocución que supuso un antes y un después tanto para los estudios literarios, como para los filosóficos, así como también para los entonces recientemente acuñados e institucionalizados estudios culturales, proclamaron la muerte del autor tras preguntarse por su naturaleza y por su estatuto en el discurso y para con la obra.

Es preciso recordar, a modo de genealogía telegráfica, que cuando menos dos pensadores contemporáneos hacen las veces ya en el siglo XIX de antecedente de dicha muerte. El primero es Kierkegaard. Cabe señalar, no por ser únicos pero sí por resultar fundamentales para el asunto en cuestión, dos aspectos destacables de su quehacer discursivo que nos permiten observar de qué modo se pone en obra en ese mismo quehacer la defenestración que nos ocupa. El primer aspecto es de carácter general en la medida en que se conforma como estrategia discursiva que se concreta a lo largo y ancho de su obra, atravesándola, en la red de pseudónimos por fuerza de la cual se fragmenta $\mathrm{y}$, de ese modo, se destituye la única voz y, entonces, el único referente para el sentido ${ }^{1}$. El sentido y, por ende, la obra que aloja el sentido, deja de estar en manos de un único propietario; el sentido y la obra son literalmente expropiados. El segundo aspecto adquiere un carácter particular en la obra escrita en 1848, aunque publicada de forma íntegra y póstuma en 1859, El punto de vista sobre mi producción como escritor. Aquí, Kierkegaard ofrece una suerte de autobiografía literaria que narra, a la par, en primera y en tercera persona, en la primera y segunda parte del texto respectivamente, deconstruyendo el género autobiográfico mediante el desplazamiento del centro de la narración del yo que garantiza no sólo el sentido de lo narrado, sino también la veracidad de ese sentido. De esa forma, Kierkegaard remite la narración de lo propio a la impropiedad narrativa. Ambos aspectos

\footnotetext{
${ }^{1}$ Kierkegaard constituye en «¿Qué es un autor?», texto que da lugar a la reflexión acerca de la figura autorial en este artículo, un referente explícito para Foucault precisamente en lo que atañe a la pseudonimia. Escribe Foucault: «[p]odríamos también interrogarnos sobre el sentido y el funcionamiento de una proposición como [...] "Victor Eremita, Climacus, Anticlimacus, Frater Taciturnus, Constantin Constantius son Kierkegaard”» (Foucault, 1969: 13).
} 
obligan a emplazar al autor en la obra de Kierkegaard en un lugar fronterizo constituido en y por la permanente desapropiación del sentido y de la veracidad del sentido, así como también de la obra. En efecto, el autor es desprovisto de su autoridad. El autor es desautorizado qua autor. Y ello, desde la obra misma, en la medida en que, lo que ahí se pone en obra no es sino dicha desautorización.

El segundo pensador que contribuye a trazar la senda que condujo en los años sesenta a la muerte del autor es, qué duda cabe, Nietzsche. Con la sonada muerte de Dios, Nietzsche da al traste con el paradigma ontológico de la substancia y/o esencia y del sujeto. Pero también, como señala Jacques Derrida en su Otobiografías. La enseñanza de Nietzsche y la política del nombre propio $(1984)^{2}$, en la lectura que ahí ofrece del prefacio de Nietzsche a Ecce Homo, da al traste con el paradigma de la enunciación qua tributo a la veracidad del sentido, puesto que la enunciación es en el corpus de Nietzsche, disimulación y, en concreto, disimulación de la línea divisoria entre el cuerpo de la obra y el cuerpo del autor. La enunciación se traza en y a través de esa línea divisoria que no es una línea fija y determinada, sino en constante transformación, móvil y fluida, en constante tensión. La enunciación se constituye de esa forma en una frontera (o, como afirmará Foucault en «¿Qué es un autor?», en un borde) en el que se ve comprometida no sólo la distinción entre el cuerpo y la obra del autor, sino también la distinción entre la vida y la muerte del autor. E incluso, cabe arriesgar, la distinción entre el autor vivo y el autor muerto.

La crítica de la que se hacen eco Barthes (1968) y Foucault (1969) y que formulan de forma explícita en sendos textos, «La muerte del autor»y «QQué es un autor?», se dirige, en complicidad con lo expuesto, a un lugar de enunciación que acapara la significación, dominándola, y se hace con la autoridad del sentido, sellándolo. Pero su crítica se dirige también a un lugar político que detenta el poder de todo cuanto es en tanto que todo lo que se enuncia, mediante el control de palabras y cosas y desde el supuesto e imposición a la vez de una relación de identidad entre ambas. Dicha crítica, ya sea en su declinación linguiística, ya sea en su declinación ontológica, tras lo considerado desde Kierkegaard y desde Nietzsche, invita a ser revisitada para observarla vis-à-vis lo que, con Deleuze y Derrida, llamamos espectro ${ }^{3}$.

\footnotetext{
${ }^{2}$ Huelga decir que Nietzsche es también en este asunto un referente primero para Foucault, quien conjuga, si bien sólo apuntándola de forma explícita, su revisión de la muerte del autor con la de la muerte del sujeto. Así lo afirma en «¿Qué es un autor?»: «[e]l autor -o lo que he tratado de definir como función-autor- sin duda no es más que una de las especificaciones posibles de la función-sujeto» (1969: 29). Lidiar (con) el fantasma del autor comporta, entonces, lidiar con el fantasma del sujeto. De ahí, en parte, la resistencia dentro y fuera del pensamiento a borrar la figura autorial. Los motivos de esa resistencia los atribuye Foucault a lo que, en la ampliación que sufrió su contribución primera con motivo de la conferencia que impartió a ese mismo respecto en la Universidad de Buffalo en 1970 y que se publicó nueve años más tarde, él mismo denominó «economía del autor»-y que permite ser considerada en paralelo a lo que identificaríamos entonces como «economía del sujeto»- en tanto que «figura ideológica mediante la que se conjura la proliferación del sentido» (1969: 30). Y, ello, a pesar de que el autor, insiste Foucault, «no es una fuente de significaciones que se colmarían en la obra [dado que] el autor no precede a las obras» (1969: 29).

${ }^{3}$ Es preciso señalar que Foucault plantea como objetivo general del texto identificar los lugares donde, a pesar de su «desaparición», el autor ejerce aún su función: «La desaparición del autor se ha convertido para la crítica en un tema ya cotidiano. Pero lo esencial no es constatar una vez más su desaparición; hay que repetir, como lugar vacío -a la vez indiferente y coactivo- los emplazamientos en donde se ejerce esta función» (1969: 2). De ahí que el texto de Foucault comporta ya un giro hacia lo espectral con relación a lo planteado y asumido desde su precedente cercano en el texto de Barthes, en la medida en que, si bien Foucault toma dicho precedente como punto de partida, se empeña en matizarlo siguiendo el rastro indeterminado e incierto del autor en sus diversas figuraciones (el nombre, la relación de atribución, la
} 
A este respecto, propongo formular las siguientes preguntas: 1) ¿en qué medida cabe considerar al autor en clave contemporánea como espectro o, dicho de otro modo, como ejemplar puesta en obra de lo espectral? O: 2) ¿qué consecuencias tiene dicha consideración para con la obra en la medida en que ésta se constituye a modo de relación que comporta un modo singular de subjetivación? Y también: 3) ¿cómo se da el sentido en esa relación si no como acontecimiento, es decir, como suspensión indefinida de la lógica causal y de cualquier modo de representación determinado por ésta?

Veamos cada una de estas preguntas.

1. ¿En qué medida cabe considerar al autor en clave contemporánea como espectro o, dicho de otro modo, como ejemplar puesta en obra de lo espectral? Esta primera pregunta también lee: ¿en qué medida cabe establecer una relación de ejemplaridad entre la figura de lo autorial y la figura de lo espectral? Es decir, ¿en qué medida cabe considerar la relación entre el autor y el espectro como prototípica, como ejemplar original a partir del cual se figuran otros ejemplares, es decir, otras relaciones que son relaciones representativas o, dicho de otro modo, relaciones dadas a la representación?

Foucault plantea ya esa misma cuestión en su texto de 1969, editado y reeditado en varias ocasiones en las dos décadas posteriores a esa fecha, «¿Qué es un autor?» ${ }^{4}$. Sin duda, no plantea la pregunta en los términos propuestos, es decir, en alusión al espectro o a la puesta en obra de lo espectral. De lo que se trata, afirmará, es de «quitarle al sujeto (o a su substituto) su papel de fundamento originario, y analizarlo como una función variable y compleja del discurso» (1969: 29). Foucault no sólo observa en las diversas configuraciones autoriales la pervivencia del autor, sino que diagnostica la resistencia del pensamiento a la borradura definitiva de su existencia. Dicha resistencia la atribuye Foucault a lo que denomina función autor en la medida en que le atribuye un ámbito de acción que excede a la obra que el autor firma y que suscita y determina a la vez dos tipos de relación con la misma: de apropiación y de atribución. La puesta en obra de la obra comporta ser puesta como tal en manos ajenas y en ello radica, más que la muerte del autor, la enajenación de la obra y de la relación de propiedad que el autor ha mantenido hasta entonces con ella. A esa puesta en obra de la obra, que es, entonces, puesta más allá de sus confines no sólo materiales, en tanto que contextuales, es decir, geográficos, temporales, etc. sino que es puesta también más allá de sus propias posibilidades de significación mientras permanece en manos del autor es a lo que Foucault denomina «instauración de discursividad» (1969: 24).

relación de atribución y la posición). Todas las figuraciones adquieren un carácter que Foucault advierte como tensional. Ese carácter tensional imposibilita la renuncia definitiva en y desde el pensamiento a la figura autorial o, aún, a la configuración autorial en tanto que «existencia» del autor.

${ }^{4}$ «Qu' est-ce qu’ un auteur?» se encuentra en Dits et écrits. 1954-1988 (París, Gallimard, Vol. 1, 1954-1969), pero se publica inicialmente en Bulletin de la Société Francaise de Philosophie (63/3, julio-septiembre de 1969, pp. 73-104). Y también se encuentra en versión inglesa en: Harari, J. V. (ed.) (1979): Textual Strategies. Perspectives in PostStructuralist Criticism. Ithaca, Cornell University Press, pp. 141-160. 
Lo que se instaura en ese excedente de la firma del autor y en la obra, entonces, concebida como excedente, especifica, no es tanto un modelo discursivo, un ejemplo al fin, que permite ser reiterado con remisión a los elementos que lo constituyen en calidad de enclaves primeros de significación, es decir, un modelo discursivo que reverbera en virtud de la analogía -como sucede, por ejemplo, con la instauración de modelos literarios-, sino que se trata, según Foucault, de modelos discursivos que permiten ser reiterados en virtud de la diferencia. En concreto, estos modelos -Foucault se refiere a Freud y a Marx- «abrieron el espacio a algo diferente de ellos que, sin embargo, pertenece a lo que fundaron»y, así, «establecieron una posibilidad indefinida de discursos» (1969: 22). Se trata, por tanto, de modelos que difieren.

A su vez, a diferencia del modelo científico, que admite la reiteración hasta el punto de poder ser reconsiderado y corregido, el modelo discursivo - que excede dicho modelo científico- al que se refiere Foucault, quien pone como ejemplo a Freud y al psicoanálisis, es «heterogéne[o] a sus transformaciones exteriores» (1969: 24). E insiste: «[a] diferencia de la fundación de una ciencia, la instauración discursiva no forma parte de sus transformaciones ulteriores, sino que permanece necesariamente retirada o sobrevolándola» (1969: 24). No hay, entonces, corrección de la puesta en obra originaria, si bien hay permanente retorno a la misma, es decir, retorno a lo que Foucault califica de «coordenadas primeras». Y este retorno permanente a la instauración discursiva «forma parte del discurso mismo [y] no deja de modificarlo», aunque no como «suplemento histórico» sino como «trabajo efectivo y necesario de transformación de la discursividad misma» 5 .

Es preciso recabar aquí en el comentario marginal que aporta Foucault al respecto de ese trabajo de transformación de la discursividad y del supuesto que dicho trabajo recae en la función autor. Lejos de dicho supuesto, es decir, como revisión crítica de dicho supuesto que comporta, a su vez, la revisión crítica de lo que Foucault llama función-sujeto, se plantea, entre paréntesis, que «[e]s posible imaginar una cultura en la que los discursos circularan y fueran recibidos sin que la función autor apareciera nunca» (1969: 29). Es decir, una cultura que, a modo de autor, dada su desaparición permanente, es decir, dada su permanencia en la desaparición, consistiera, precisamente, en lo que he propuesto llamar ejemplar puesta en obra de lo espectral, atendiendo a ella como condición necesaria de toda obra.

Y así avanzamos hacia la segunda pregunta anunciada.

2. ¿Qué consecuencias tiene considerar al autor como espectro o como ejemplar puesta en obra de lo espectral para con la obra en la medida en que ésta se constituye a modo de relación que comporta un modo singular de subjetivación? Esta pregunta toma en consideración otra que la

\footnotetext{
${ }^{5}$ El texto «¿Qué es un autor?» es, de hecho, un ejemplo de dicha puesta en obra de la obra como excedente, de dicha transformación de la discursividad misma en la medida en que, como afirma Foucault en su preámbulo, dicho texto comporta un retorno a las «coordenadas primeras» que, al respecto de la figura autorial, ofrece Las palabras y las cosas para plantear una crítica que se estima necesaria. Este retorno tiene como objetivo explícito «volver sobre un cierto número de imprudencias que llegué a cometer» (1969: 4) afirma Foucault, y definir cómo se utiliza «el nombre de autores» (1969: 5).
} 
antecede y que la informa, relativa al estatuto de la obra, y que Foucault formula en los siguientes, sin duda afines términos: «[e]ntre los millones de huellas que alguien deja después de su muerte ¿cómo puede definirse la obra?» (1969: 9). Y añade, advirtiendo de la complejidad de atribución de un estatuto propio a la obra: «[r]esulta insuficiente afirmar: prescindamos del escritor, prescindamos del autor y vayamos a estudiar la obra en sí misma. La palabra "obra” y la unidad que designa son, probablemente, tan problemáticas como la individualidad del autor» (1969: 9).

Pero, volviendo a la pregunta planteada, es preciso atender a dos circunstancias que, a mi entender, condicionan la obra en tanto que relación y/o consiguiente modo de subjetivación.

1) En primer lugar, es preciso recordar lo que ya ha sido elaborado en lo anterior, a saber, que la instauración discursiva, es decir, las «coordenadas primeras» en que cabe inscribir toda obra, tiene un carácter de «retirada» o de «sobrevuelo» y eso es constitutivo de todo retorno a las mismas. No se retorna, por tanto, a suelo firme. A este respecto afirma Foucault que «la palabra "obra" y la unidad que designa probablemente son tan problemáticas como la individualidad del autor» (1969: 9).

El retorno en que consiste, entonces, la instauración discursiva, en tanto que retorno a la obra en tanto que retorno a las coordenadas primeras, considera Foucault: «se dirige a lo que está presente en el texto, más precisamente se regresa al texto mismo, al texto en su desnudez y, a la vez, sin embargo, se regresa a lo que está marcado en hueco, en ausencia, como laguna en el texto. Se regresa a un cierto vacío que el olvido ha esquivado o enmascarado, que ha recubierto con una falsa plenitud, y el retorno debe redescubrir esta laguna y esta carencia» (1969: 26). No cabe duda que el retorno de la instauración discursiva lleva la marca del nietzscheano método genealógico. Es decir, que la obra se constituye en el retorno en la medida en que éste se conforma como relación cuyo objetivo es doble. Por un lado, visibilizar los vacíos que constituyen a la obra y, por otro, visibilizar las estrategias por medio de las cuales se ha procedido a olvidarlos.

2) La segunda circunstancia que condiciona la obra en tanto que relación y/o consiguiente modo de subjetivación conduce a una segunda elaboración que lleva a cabo Foucault al respecto de la desaparición del autor y que remite a una noción clave en el asunto que nos ocupa. Escribe Foucault: «[o]tra noción retiene al pensamiento al borde de la desaparición del autor [...]. Es la noción de escritura. Rigurosamente, debería permitir no sólo prescindir de la referencia al autor, sino darle estatuto a su nueva ausencia» (1969: 10). ¿A qué se refiere Foucault con nueva ausencia? Y, además, ¿qué motiva el reclamo de otorgar estatuto a esa nueva ausencia? Aclara Foucault: «[p]ensar la escritura como ausencia ¿no es simplemente repetir en términos transcendentales el principio religioso de la tradición a la vez inalterable y siempre llena, y el principio estético de la supervivencia de la obra, de su conservación más allá de la muerte y de su exceso enigmático con respecto al autor?» (1969: 10). Foucault indica el riesgo que conlleva identificar la desaparición del autor con su ausencia tout court y reclama un estatuto para el mismo que evite relegarlo unilateralmente y, así, unívocamente, ya al cielo ya a la tierra. El autor se ve emplazado, entonces, como lo hiciera la enunciación, a un lugar intermedio que va a determinar las relaciones con la obra y, así, la instauración discursiva misma como interés qua inter-esse, es decir, como «ser entre». El 


\section{Begonya Sáez Tajafuerce}

estatuto de la nueva ausencia del autor radica en esa determinación intermedia como lugar (im)propio de una economía otra. Así como la obra deviene excedente, el autor deviene espectro. Para ambos casos rige la ejemplar puesta en obra de lo espectral.

En tanto que desaparecido, el autor no está, al decir de Derrida en una entrevista sobre cine ${ }^{6}$ en la que plantea el carácter y, a la vez, la fuerza espectral de la imagen en ese contexto, ni «vivo ni muerto». Y, por si eso fuera poco, el autor, en complicidad con la instauración discursiva, y, entonces, también en calidad de coordenada primera, en los mismos términos en que lo hiciera la obra, se retira y sobrevuela. De ahí, también, que resulte pertinente plantear esa nueva ausencia del autor en términos de ejemplar puesta en obra de lo espectral. Como espectro, el autor aparece al desaparecer. Además, el espectro, dice Derrida, como el autor, por otra parte, «es un enigma» en el cual cabe creer, pero dicha creencia no constituye prueba alguna, no es, por tanto, fuente de veracidad ni asegura vínculo alguno con verdad alguna. Lo espectral, afirma Derrida: «es un elemento [...] en el que la creencia no es asegurada ni desmentida».

En efecto, la relación ante la que nos encontramos poco o nada tiene que ver con la relación a que da lugar el modo de discursividad científico o su autor, ya sea vivo o muerto. La condición de singularidad de la discursividad de la obra, así como la condición de singularidad del autor, determina la relación en que se constituye la obra como relación singular. Al respecto, escribe Foucault: «[1] os campos discursivos de los que hablo conllevan respecto de su autor "fundamental" y mediato una relación que no es idéntica a la relación que un texto cualquiera mantiene con su autor inmediato» (1969: 27). El texto (se) establece (en) una relación con el autor que ya no es «esa figura que le es exterior y anterior» (1969: 6), que ya no es, en efecto, el autor inmediato, es decir, el autor que se le supone.

$\mathrm{Si}$ acordamos que la singularidad de ese autor fundamental y mediato, que permite diferenciarlo del autor inmediato, radica en su carácter espectral, cabe preguntar en qué consiste, entonces, la relación con el espectro o en qué consiste, pues, la lógica de lo espectral en este contexto relacional. $\mathrm{O}$, dicho de otro modo, ¿en dónde radica la singularidad de esa relación de modo que no sea idéntica «a la relación que un texto cualquiera mantiene con su autor inmediato»? Y, aún ¿en dónde radica la singularidad de esa relación a que da lugar la obra en tanto que instauración discursiva con su autor-espectro?

\footnotetext{
${ }^{6}$ Se trata de la entrevista que realizaran Antoine de Baecque y Thierry Jousse y que se publicó con el título de «El cine y sus fantasmas» (2001). Derrida sugiere en dicha entrevista un nuevo ámbito de investigación al que da lugar la experiencia cinematográfica en tanto que experiencia de lo espectral y que, a mi entender, entronca con el reclamo manifestado por Foucault de atorgar estatuto a la nueva ausencia del autor. Cuenta Derrida: «Si escribiera sobre el cine, lo que me interesaría sería sobre todo su modo y su régimen de creencia. Hay en el cine una modalidad del creer absolutamente singular: hace ya un siglo se inventó una experiencia sin precedentes de la creencia. Sería apasionante analizar el régimen del crédito en todas las artes: cómo se cree en una novela, en ciertos momentos de una representación teatral, en lo que está inscripto en la pintura, y, por supuesto -algo totalmente diferente- en lo que el cine nos muestra y nos relata». Pensar la escritura como ausencia comporta también la pregunta relativa al estatuto de la creencia en la medida en que el autor en tanto que función-autor en la nueva ausencia que señala Foucault, es decir, en su permanencia en la desaparición, declina e imposibilita establecer y sostener un régimen de veracidad como el que parece posible rescatar todavía en y para el modelo científico.
} 
3. Estas preguntas nos conducen a la tercera planteada al inicio, a saber: ¿cómo se da el sentido en la relación de la obra con su autor-espectro si no como acontecimiento, es decir, como suspensión indefinida de la lógica causal y de cualquier modo de representación determinado por ésta? Recordemos que el autor-espectro ha sido emplazado a un lugar intermedio para el que rige una economía otra. Dicho emplazamiento y dicha economía suspenden la lógica causal y cualquier modo de representación determinado por ésta. Dicha suspensión (in)determina el sentido en la medida en que éste deviene tan sólo en la relación singular de la obra con el autor en los términos descritos -en lugar de devenir en una relación de carácter general y con validez universal-. Esa relación singular da lugar al sentido. Y aquí resulta clave el dar lugar. En ello radica el singular modo de darse el sentido, que no es el modo que corresponde a la lógica causal, por fuerza de la cual el sentido se da de antemano, se presupone así como se presupone el autor, la obra y la relación entre ambos, sino a esa economía otra que suspende esa lógica causal. Pensar la escritura desde la ausencia comporta el compromiso del sentido en ese (tener) lugar que la reflexión contemporánea en torno a la representación se ha dado en llamar acontecimiento. El sentido, en esa relación singular de la obra con el autor-espectro, a tenor de la economía de lo espectral, entonces, acontece.

Lo que se plantea aquí concierne, en definitiva, al estatuto de la representación en el contexto del pensamiento contemporáneo y a la tarea asumida de pensar, con Deleuze, «fuera de la representación en donde toda diferencia se somete al principio de identidad».

Hemos visto en qué medida se resiste el principio de identidad cuando el retorno a la instauración discursiva no comporta un retorno a suelo firme alguno y cuando el autor «fundamental y mediato» es un espectro. En ambos casos, la relación para con la obra se pliega a los parámetros de una economía que no se somete a ningún principio universal tal que el principio de no contradicción. Es por ello que la relación para con la obra sucede, en términos de Deleuze en La lógica del sentido ${ }^{7}$ a la par como «resultado de acción y de pasión» (1969: 248). No hay referente último que dirima en esa dialéctica indiscernible, puesto que, el autor en tanto que garante de la relación, del sentido y de la veracidad del mismo, «el comienzo está verdaderamente en el vacío, suspendido en el vacío. El comienzo es with-out. La situación paradójica del comienzo, aquí, es que es en sí mismo un resultado, por una parte, y por otra, permanece exterior a lo que hace comenzar» (1969: 257). Y ello se debe al hecho de que, el yo, el individuo que ya defenestrara Nietzsche y el sujeto que

\footnotetext{
${ }^{7}$ Voy a citar de la trigésima serie de paradojas: «El fantasma». Indica Miguel Morey en la introducción de la edición en castellano que este texto de Foucault se articula según dos vectores principales: el lógico y el psicoanalítico. El primer vector traza la línea de lo que denomina «sentido-acontecimiento» (2011: 18) y, el segundo, la línea del «sentidofantasma» (ídem). Y augura: «Preguntarse así por el sentido es preguntarse por un incorporal que sobrevuela todos los estados de cosas, por un extraser que frente a las alturas del ideal o la física de las profundidades se mueve en una superficie metafísica [...] Preguntarse así el sentido no es sino pensar el acontecimiento: ese morir que pasa y se hace muerte, esa muerte que hace presente el problema eterno del morir» (2011: 19). El problema central para Deleuze, indica Morey, en Lógica del sentido, es el pasar en cuanto tal. Y ese problema se articula en una doble pregunta: «¿qué es lo que pasa?» $\mathrm{y}$ «¿̨en qué sentido?» (2011: 20). Esa doble pregunta pone en obra el pensamiento en tanto que "un modo de producir sentido operando casi en el vacío" (ídem). De ahí que dicha articulación constituya por sí misma una propuesta «fuera y contra toda representación» (2011: 21).
} 
reconsiderara Foucault, deja de operar como causa por no ser «ni activo ni pasivo»y por «no dejarse fijar [...] en ningún momento [...] en ningún lugar» (1969: 250).

Así, la relación sucede en sentido estricto. Es, pues, acontecimiento. Y, en tanto que acontecimiento, efecto. Y así también el sentido. Pero, según Deleuze, se trata de un efecto singular que responde a lo que identifica como una «doble causalidad» (1969: 249), en virtud de la cual, por un lado, el acontecimiento -y, con él, pues, el sentido- sucede en el espacio intermedio o fronterizo en el que participan «unas causas no sólo endógenas, sino también exógenas» (1969: 248) y, por otro, «está sometido [...] a las causas externas e internas de las que resulta en profundidad» (ídem). Pero, también, el acontecimiento no es «ni imaginario ni real» (ídem). De ese modo, la relación con la obra «nos inspira una espera insoportable, la espera de lo que va a resultar, de lo que está ocurriendo y no acaba de ocurrir» (ídem). Ésa es la lógica otra del acontecimiento que, sin duda, propone otra formulación tanto de la «instauración discursiva» de Foucault como del diferir del sentido siempre enigmático de Derrida, haciéndose eco del desplazamiento que llevara tanto a la enunciación como al autor-espectro a un lugar intermedio. Desde y para el inter-esse, la relación y, con ella, el sentido, suceden, en efecto, entre el cielo y la tierra.

\section{Referencias bibliográficas}

BARTHES, R. (1968): «La muerte del autor», en El susurro del lenguaje. Barcelona, Paidós, 1987, pp. 65-71.

DeleuZe, G. (1969): Lógica del sentido. Madrid, Paidós, 2011.

DERRIDA, J. (1984): Otobiografías. La enseñanza de Nietzsche y la política del nombre propio. Buenos Aires, Amorrortu, 2009.

- (2001): «El cine y sus fantasmas», en A. de Baecque y T. Jousse, eds., Cahiers du cinéma, 556.

FOUCAULT, M. (1969): «¿Qué es un autor?», en http://www.elseminario.com.ar/biblioteca/Foucault_ Que autor.htm. 